\title{
Low Frequency Axial Flux Linear Oscillating Electric Drive Suitable for Short Strokes
}

\author{
Govindaraj Thangavel \\ Department of Electrical \& Electronics Engineering, Muthayammal Engineering College, Rasipuram 637 408, India \\ Correspondence should be addressed to Govindaraj Thangavel; govindarajthangavel@gmail.com
}

Received 23 September 2013; Accepted 20 October 2013; Published 30 January 2014

Academic Editors: M. Hopkinson and R. Newcomb

Copyright (C) 2014 Govindaraj Thangavel. This is an open access article distributed under the Creative Commons Attribution License, which permits unrestricted use, distribution, and reproduction in any medium, provided the original work is properly cited.

\begin{abstract}
The design, analysis, and control methodology of an energy efficient and high force to weight ratio rare earth N42 NdFeB based permanent magnet linear oscillating motor has been described. For this axial flux machine the mover is consisting of Aluminium structure embedded with rare earth permanent magnets of high energy density. Microcontroller based drive is developed for frequency and thrust control of the machine. Finite element method using FEMM is employed for analysis of various performance parameters of machine. The same parameters are also compared with the measured ones, which yields a good agreement to the proposed design.
\end{abstract}

\section{Introduction}

Permanent magnet linear oscillating motors (PMLOMs) are finding increased suitability for many applications [13]. These motors require accurate oscillating/reciprocating characteristics for high precision application. Also the power requirements for these motors play an important role from efficiency point of view. The use of linear reluctance motors is already studied [2] under alternating current and also with direct current supply $[3,4]$. The d.c. motors are having negligible core losses and therefore show higher efficiency than the a.c. ones, although the a.c. motors are employed in many applications [3]. The a.c. motors are used in pumps and many linear actuators [5]. The linear oscillating motors (LOMs) differ in terms and technologies as well as construction [3] from their rotating counterparts. The motors with the small oscillating frequency but high stroke length are used as a shuttle power drive for looms (in the textile industry) or as electric hammers. In $[1,3]$ the different applications for low frequency operation are given. In $[6,7]$ magnetic field analysis for tubular motors has been presented for mostly flat induction motors. In $[5,8,9]$ the field circuit models have also been applied for simulation of dynamic characteristics of the tubular motors. However, the investigations did not include the permanent magnet axial flux type linear oscillating motors (PMLOMs). The short stroke oscillators are mainly applicable in water pumps. These motors work relatively at higher frequencies. The proposed motor as given in this paper is suitable for short stroke and low frequency application from 0 to $5 \mathrm{~Hz}$. This machine has applicability for the development of heart pump with adjustable stroke frequency and thrust force.

In the present work, the field calculations of the PMLOMs and their speed and thrust control techniques are presented. In formulated motion and electrical circuit equations, the calculated parameters are used. A PMLOM is a device, which directly uses the forces of attraction and repulsion between a permanent magnet and an electromagnet. The main structure of the motor is shown in Figure 1. The proposed motor can easily be powered and controlled by a small, portable microcontroller based power system. It has a high forcedensity, high efficiency, and smaller size and weight. It can satisfy the performance requirements with a variable stroke volume and beat rate control. Suitable simulations and experiments validate the proposed concept.

\section{Permanent Magnet Linear Oscillating Motor (PMLOM)}

2.1. Principle of Oscillating Motion. PM attraction/repulsion type linear motor is shown in Figure 1, from which it follows 


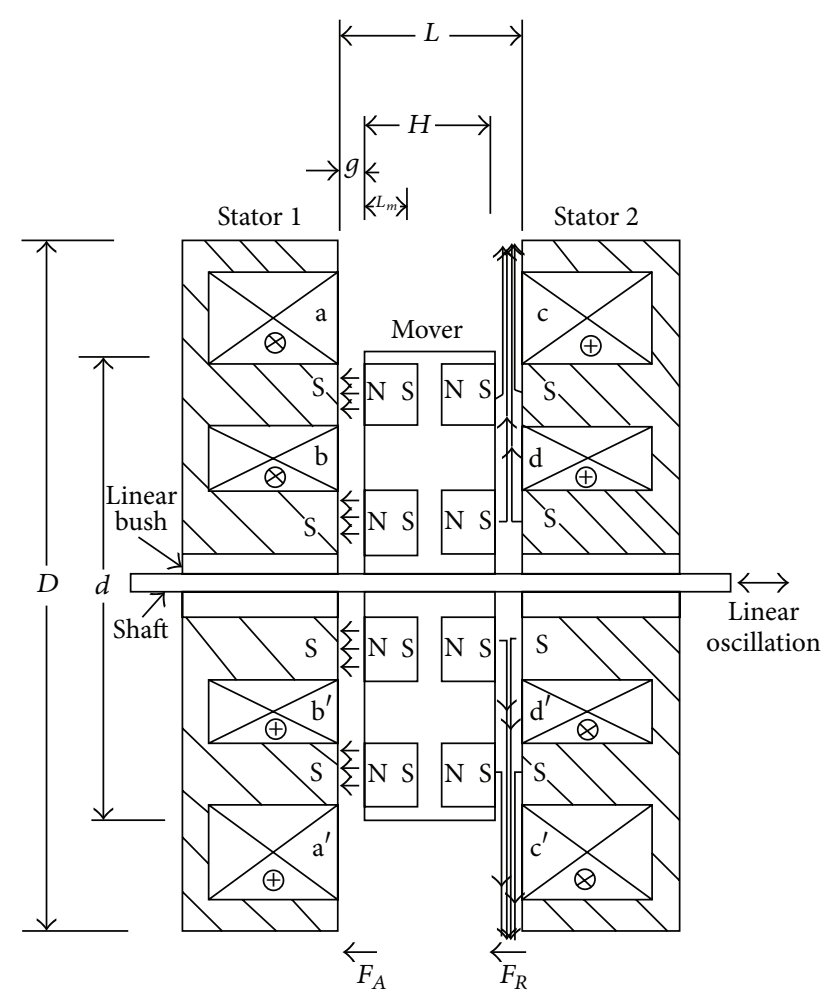

FIGURE 1: Principle of operation of the PMLOM attraction force and repulsion force.

that the motor is a controlled magnet system and has a variable axial airgap. Several topological variations of the electromagnetic structures are feasible. The proposed motor has a cylindrical structure with two stators, each having two slot-embedded coils (coil 1 in stator 1 consists of coils aa' and $\mathrm{bb}^{\prime}$ and coil 2 in stator 2 consists of coils $\mathrm{cc}^{\prime}$ and $\mathrm{dd}^{\prime}$ ) and a mover with ring type rare earth permanent magnets. For the relative polarities of the currents in the various coils it may be readily verified that there will be an attraction force between coil 1 and the permanent magnets and a repulsion force between coil 2 and the permanent magnets. Thus, the mover will tend to move to the left. At the end of the stroke, the polarities of the currents in coils 1 and 2 are simultaneously reversed. This reversal changes the directions of the forces. A repulsion force now exists between coil 1 and the permanent magnets and an attraction force exists between coil 2 and the permanent magnets. Consequently, the mover tends to move to the right. At the end of the stroke the polarities of the currents in coils 1 and 2 are reversed again and go on cyclically. Hence, sustained oscillations are obtained. PMLOMs are based on several phenomena. The fundamental is the change in magnetic energy with the mover movement. The energy is maximum when the mover is situated nearer to any one of the stators. When it is moved away from the stator, energy is lower. The energy changing produces the magnetic force, which results in the oscillating motion of the mover.

\section{Performance Analysis of PMLOM}

3.1. Attraction Force. Using the model and dimensions as given in Figure 1, the inductance of the exciting coil can be written as

$$
L=\frac{\psi}{I}=\frac{\mu_{0} N^{2} x \pi d}{2 g}
$$

where $\psi$ is flux linkage, $N$ is number of turns, $\mu_{0}=4 \pi \times$ $10^{-7} \mathrm{H} / \mathrm{m}, d$ is diameter of the mover, $I$ is current in exciting coil, $g$ is axial airgap length, and $x$ is displacement of the mover at any point of time.

From Ampere's law, neglecting the reluctance drop in the iron, the main flux density in the airgap is determined by the magnetic circuit analysis of the permanent magnet and the electromagnet. When the polarities are opposite, the MMFs of primary coil and secondary PM assist each other, but when the polarity is the same, the MMfs of primary coil and secondary PM oppose each other. Knowing coil inductance $L$ from (1), the force in terms of coil current $I$ and the variation of the inductance $L$ with the position $x$ are given by

$$
F_{A}=\frac{1}{2} I^{2} \frac{d L(x)}{d x}=\frac{\mu_{0} N^{2} I^{2} \pi d}{4 g} .
$$

It may also be shown from the basic electromagnetic field equations that the energy $W_{m}$ stored in the airgap is given by

$$
W_{m}=\frac{B_{g}^{2}}{2 \mu_{0}} 2 g x \pi d
$$

where $B_{g}$ is airgap flux density.

The electromagnetic force is obtained from

$$
F=\frac{\partial W_{m}}{\partial x}=\frac{B_{g}^{2}}{2 \mu_{0}} 2 g \pi d .
$$

In these machines the flux density, $B_{g}$, can be increased to 1 tesla without saturating the core, in which case the force density becomes $4 \times 10^{5} \mathrm{~N} / \mathrm{m}^{2}$.

3.2. Repulsion Force. Now, considering the repulsion force, we have the polarities of the currents reversed. The fluxes in the airgap are predominately radial. Qualitatively, it may be seen from Figure 1 that we now have a force of repulsion between the coil and the permanent magnets. For a small airgap and for a uniform flux density in the airgap a simple magnetic circuit approach yields the magnetic stored energy.

From Ampere's law, neglecting the reluctance drop in the iron, the flux density of coil is related to the potential (MMF) by

$$
\begin{gathered}
B_{E}=\mu_{0} H_{E}=\frac{\mu_{0} N I}{H+2 g}, \\
H_{E}=\frac{N I}{H+2 g},
\end{gathered}
$$




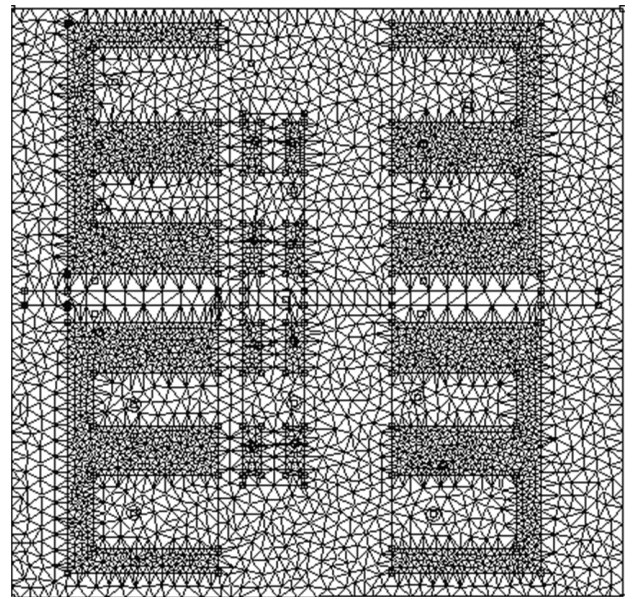

(a)


(b)

FIgURE 2: (a) Finite element mesh of PMLOM while mover is oscillating with in stator 1. (b) Magnetic flux plotting of PMLOM while mover is oscillating with in stator 1 , at $1 \mathrm{~Hz}$.

where $H_{E}$ is magnetic field intensity along radial direction and $B_{E}$ is flux density along radial direction. Referring to Figure $1, B_{E}, \phi_{E}, \psi_{E}$, and $L_{E}$ can be obtained as

$$
\begin{aligned}
& \phi_{E}=B_{E} \pi d\left(l_{s}-x\right)=\frac{\mu_{0} N I \pi d\left(l_{s}-x\right)}{H+2 g} \\
& \psi_{E}=N \phi_{E} \\
& L_{E}=\frac{\psi_{E}}{I}=\mu_{0} N^{2} \frac{\pi d\left(l_{s}-x\right)}{H+2 g},
\end{aligned}
$$

where $\phi_{E}$ is magnetic flux along radial direction, $\psi_{E}$ is magnetic flux linkage along radial direction, $L_{E}$ is inductance along radial direction, $l_{s}$ is stroke length, and $H$ is thickness of mover.

The above expression may be combined to give the repulsion force $F_{R}$ in terms of $I$ and the variation of $L_{E}$ with $x$ as

$$
F_{R}=\frac{1}{2} I^{2} \frac{\partial L_{E}}{\partial x}=\frac{\mu_{0}}{2}(N I)^{2} \frac{\pi d}{H+2 g} .
$$

The repulsion force $F_{R}$ will assist the attractive force $F_{A}$ as shown in the Figures 2(a) and 2(b). Then combining (2) and (7), the total force $F_{t}$ becomes

$$
F_{t}=F_{A}+F_{R}=\frac{\mu_{0}}{2}(N I)^{2} \pi d\left(\frac{1}{2 g}+\frac{1}{H+2 g}\right) .
$$

\section{Magnetic Field Analysis Simulation}

The proposed model was analyzed through FEMM environment, which provides the analysis through finite element method. The inductance of the stator at different frequency is calculated from the analysis. The magnetic flux density at the mover, exciting winding, and airgap region are calculated with FEM method. The calculation process involves the modeling of the geometry, setting boundary conditions and
TABLE 1: PMLOM parameters.

\begin{tabular}{lc}
\hline Rated input voltage & $70 \mathrm{~V}$ \\
Rated input power & $175 \mathrm{watts}$ \\
Stroke length & $10 \mathrm{~mm}$ \\
Outer diameter (stator) & $93 \mathrm{~mm}$ \\
Stator core type & Cold rolled grain oriented \\
silicon steel & $0.27 \mathrm{~mm}$ \\
Thickness of lamination & $30 \mathrm{~mm}$ \\
Stator length & 500 \\
Number of turns in coils aa' ${ }^{\prime}$ and $\mathrm{cc}^{\prime}$ & 250 \\
Number of turns in coils $\mathrm{bb}^{\prime}$ and $\mathrm{dd}^{\prime}$ & $14.2 \mathrm{ohms}$ \\
Coil resistance & $17 \mathrm{~mm}$ \\
Slot depth & Rare earth N42, NdFeB \\
Magnet type & $2 \mathrm{~mm}$ \\
Permanent magnet length & $975000 \mathrm{~A} / \mathrm{m}$ \\
Coercivity & $1.2 \mathrm{~T}$ \\
Remanence & $65 \mathrm{~mm}$ \\
Outer diameter (mover) & $8 \mathrm{~mm}$ \\
Shaft diameter & $0.18 \mathrm{Henry}$ \\
Coil inductance &
\end{tabular}

domain properties, generating the finite element mesh, and calculating the internal parameter at different frequency as well as at different displacement. Thus, after including the main dimensions of the machine (Figure 1) the physical properties of materials under investigation have been given. The corresponding mesh and plot of flux lines for the machine with the mover position are shown in Figures 2(a) and 2(b), respectively.

The simulation was done with dimensions and parameters of the same machine taken for experiment. The parameters are given in Table 1. 


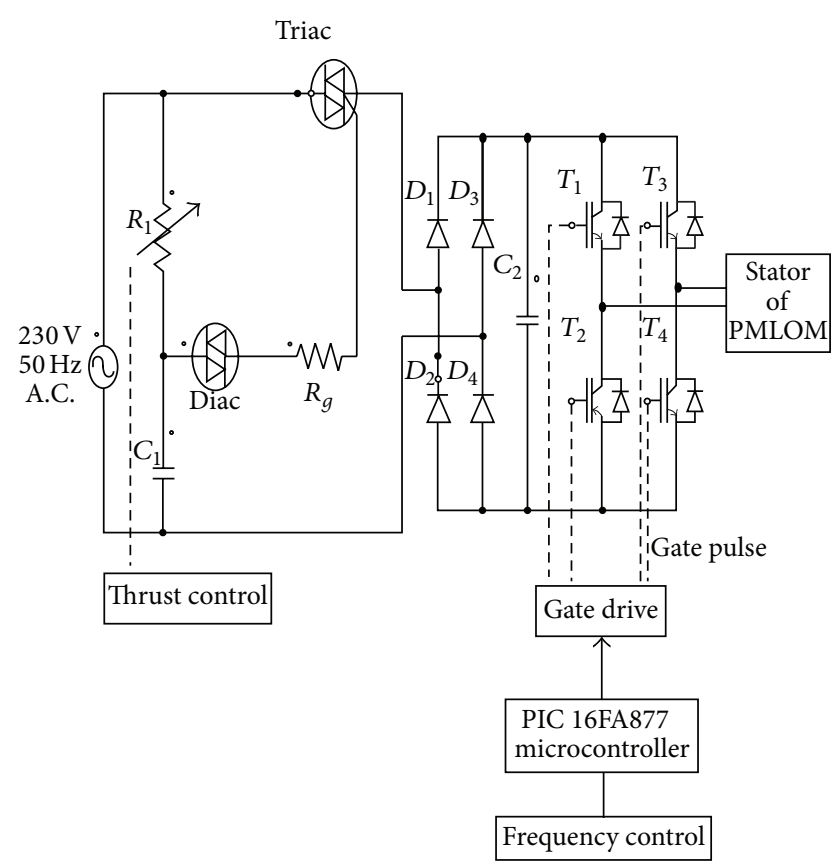

FIgURE 3: Power circuit of PMLOM.

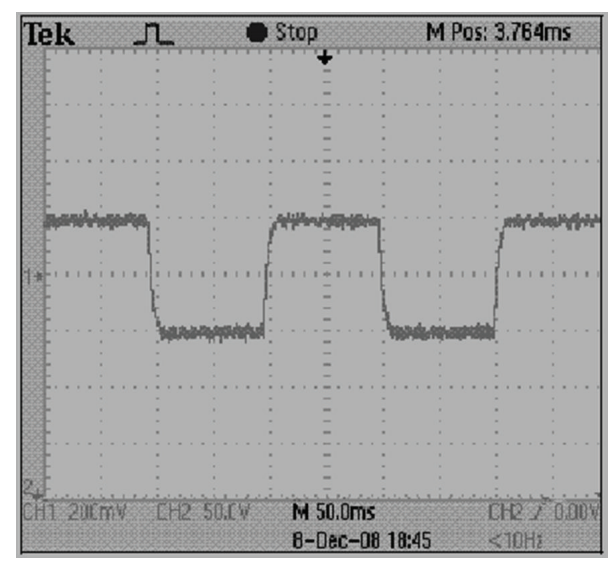

FIgURE 4: Current waveform of PMLOM taken from Tektronix make Storage oscilloscope.

\section{Experimental Results}

The machine was given a variable voltage as shown in Figure 3. The input to the motor is through an IGBT based inverter whose gate drive is controlled by a PIC16F877A digital microcontroller. The output of the PIC processor is fed to a gate driver, which ultimately supplies the gating signals. The stroke frequency can be controlled through the microprocessor whereas the thrust force can be controlled by a phase angle controller through a triac. The input current waveform is taken through a Tektronix make Storage oscilloscope and shown in Figure 4 for 2 Amps, 50 Volts supply at $5 \mathrm{~Hz}$. The force measurement is done through a force transducer and then compared with theoretical values. The axial airgap length versus total force from (8) is shown in Figure 5. The measured current versus power in watts, voltage



FIGURE 5: Airgap versus total force resultant of oscillating mover of PMLOM.

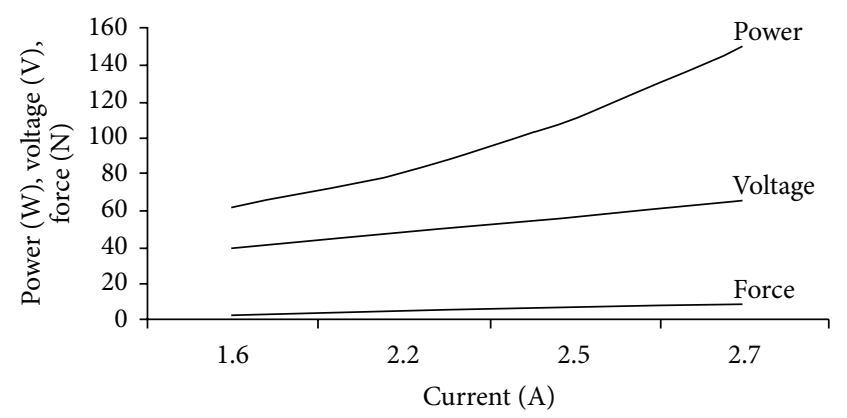

Figure 6: Measured coil current versus power (W). Voltage (V) and Force $(\mathrm{N})$ characteristics of PMLOM.

in volts, and force in Newton at $1 \mathrm{~Hz}$ frequency characteristics is plotted and shown in Figure 6.

\section{Conclusion}

Analytical solution to the forces and determination method of the integral parameters of a PMLOM are presented. Finite element method with FEMM4.2 is used for the field analysis of the different values of the exciting current and for variable mover position. Computer simulations for the magnetic field distribution and forces are given. To obtain experimentally the field distribution and its integral parameters, a physical model of the motor together with its electronic controller system has been developed and tested. The prototype has been operated in the oscillatory mode with small loads at low frequency up to $5 \mathrm{~Hz}$. The theoretically calculated results are compared with the measured ones and found a good conformity.

\section{Conflict of Interests}

The author declares that there is no conflict of interests regarding the publication of this paper. 


\section{References}

[1] I. Boldea and S. A. Nasar, Linear Motion Electromagnetic Systems, Wiley, New York, NY, USA, 1985.

[2] E. A. Mendrela, "Comparison of the performance of a linear reluctance oscillating motor operating under AC supply with one under DC supply," IEEE Transactions on Energy Conversion, vol. 14, no. 3, pp. 328-332, 1999.

[3] S. A. Nasar and I. Boldea, Linear Electric Motors, Prentice-Hall, Englewood Cliffs, NJ, USA, 1987.

[4] B. Tomczuk and M. Sobol, "Influence of the supply voltage on the dynamics of the one-phase tubular motor with reversal motion," in Proceedings of the 39th International Symposium Electrical Machines (SME '2003), pp. 417-426, Gdansk/Jurata, Poland, June 2003.

[5] N. Sadowski, R. Carlson, A. M. Beckert, and J. P. A. Bastos, "Dynamic modeling of a newly designed linear actuator using 3D edge elements analysis," IEEE Transactions on Magnetics, vol. 32, no. 3, pp. 1633-1636, 1996.

[6] M. Rizzo, "3-D finite element analysis of a linear reluctance motor by using difference scalar potential," IEEE Transactions on Magnetics, vol. 32, no. 3, pp. 1533-1536, 1996.

[7] B. Tomczuk and M. Sobol, "Analysis of tubular linear reluctance motor (TLRM) under various voltage supplying," in Proceedings of the 16 International Conference on Electrical Machines (ICEM '04), vol. 3, pp. 1099-1100, Cracow, Poland, September 2004.

[8] L. Nowak, "Dynamic FE analysis of quasiaxisymmetrical electromechanical converters," IEEE Transactions on Magnetics, vol. 30, no. 5, pp. 3268-3271, 1994.

[9] B. Tomczuk, "Three-dimensional leakage reactance calculation and magnetic field analysis for unbounded problems," IEEE Transactions on Magnetics, vol. 28, no. 4, pp. 1935-1940, 1992. 

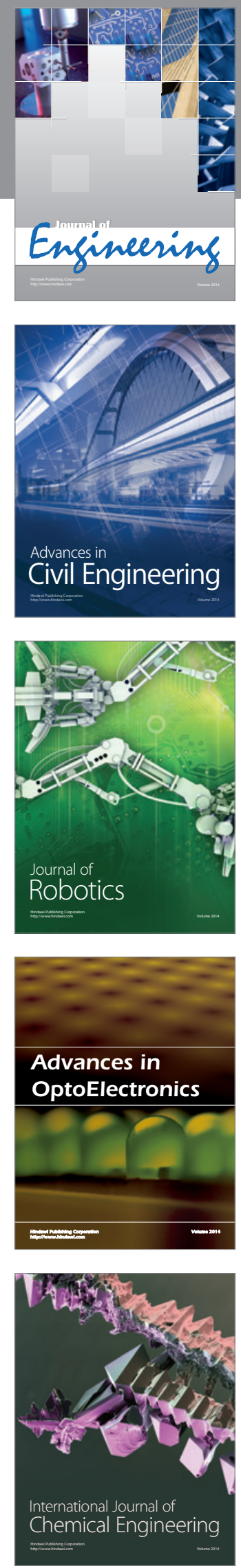

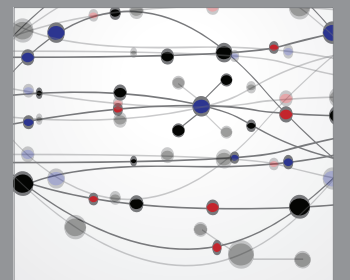

The Scientific World Journal
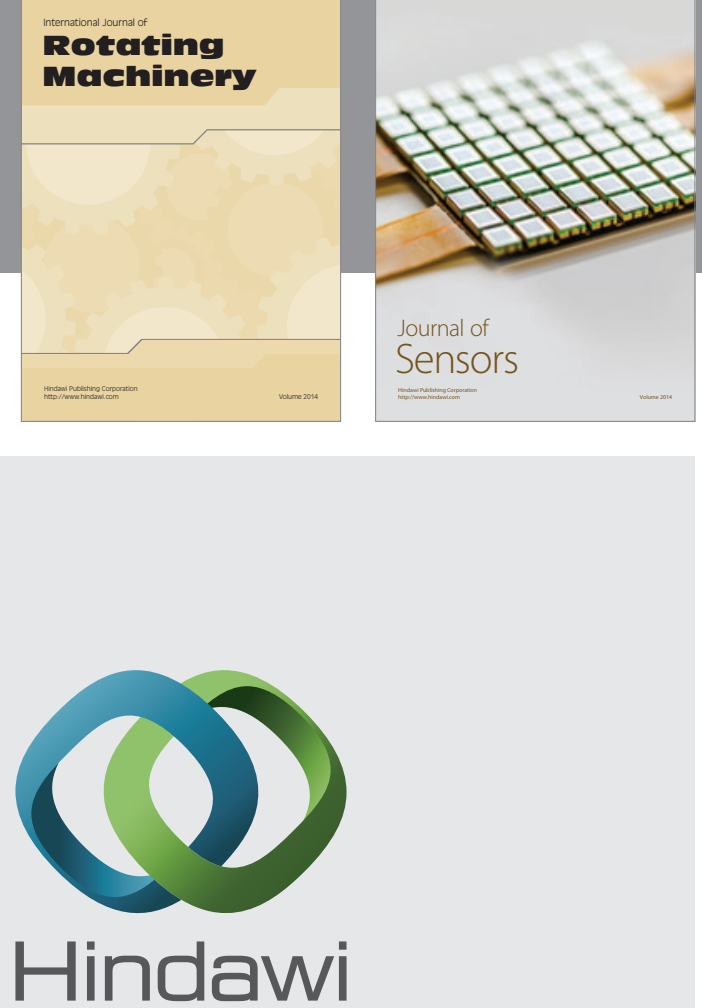

Submit your manuscripts at http://www.hindawi.com
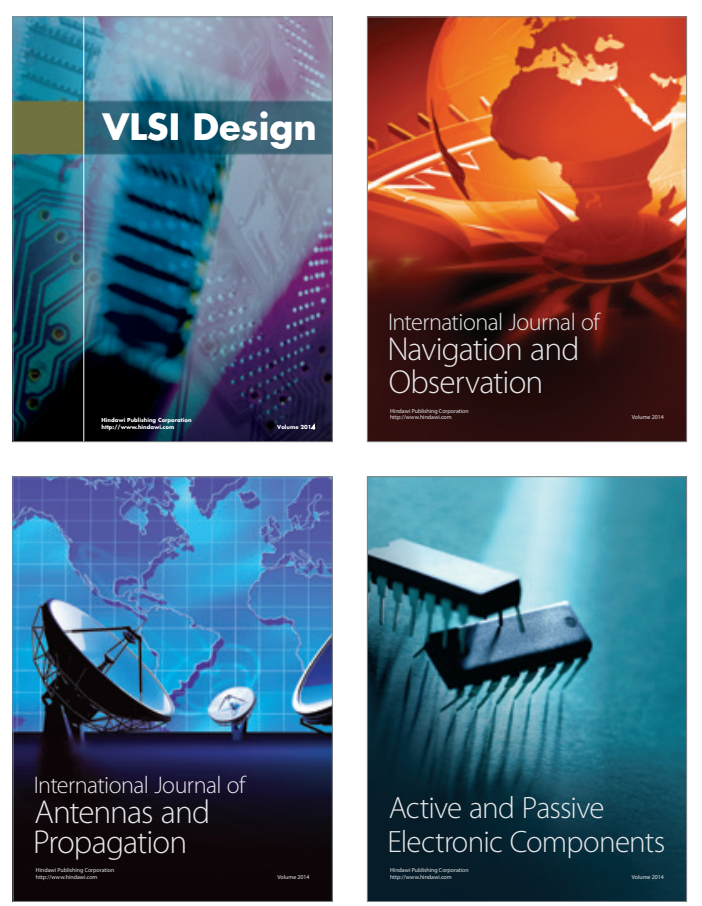


Journal of

Control Science

and Engineering
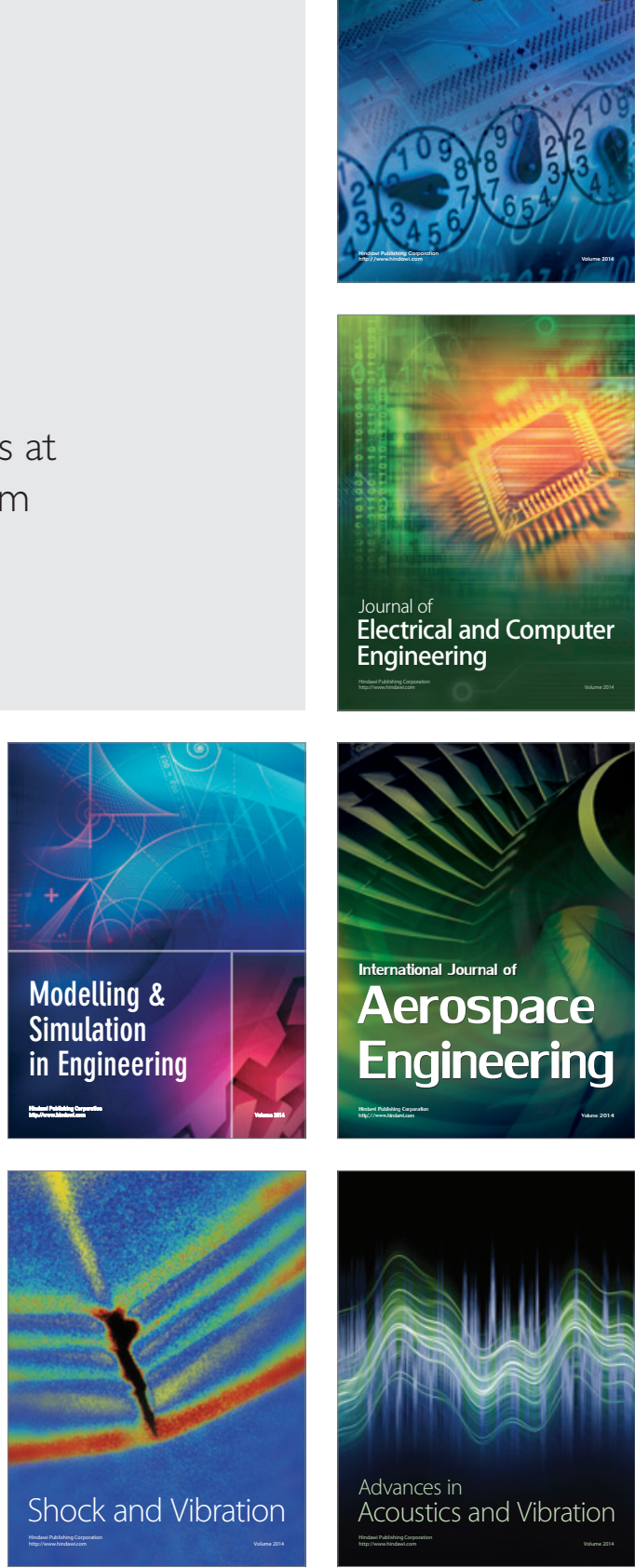